\title{
Design and Development of Distance Laboratory Package for Teaching Basic Electronics via Cloud Computing
}

\author{
https://doi.org/10.3991/ijoe.v13i08.6985 \\ Sirimonpak Suwannakhun $\left({ }^{\bowtie}\right)$, Tanes Tanitteerapan \\ King Mongkut's University of Technology Thonburi (KMUTT), Bangkok, Thailand \\ sirimonpak.sut@kmutt.ac.th
}

\begin{abstract}
This research presents the design and development of a distance laboratory package for teaching undergraduate students basic electronics via the LabVIEW program to foster more understanding through additional practice in conventional contexts rather than studying only theory in the classroom. This research consists of two main parts. The first part is the design and development of the experimental set. The subject content was analyzed to design the experiment. The competencies and skills for each topic were defined using objective analysis. Design of the experimental procedures and tools for the laboratory were implemented using task analysis. The second part is the design of the laboratory sheet on the LabVIEW program. The results revealed that students who studied with the specified experimental set had higher achievement at a significantly increasing level $(0.05)$.
\end{abstract}

Keywords - distance laboratory package, teaching basic electronics, cloud computing

\section{$1 \quad$ Introduction}

Recently, distance education has become one of the preferred choices by many people due to ease of access, availability, and integrated features: chat, forum, video conference, remote and virtual laboratories, etc. Most students expect all training materials to be available online and accessible for convenient downloading at home or during lectures. Posting lecture slides and notes as well as exercises and tests online has become common practice all over the world. This trend is also pushing universities to bring laboratory exercises to the online environment, improving on what can be considered as the next step in this process due to the higher complexity of corresponding technical solutions.

Nektarios Kostaras, Michalis Xenos and Athanassios N. Skodras [4] evaluated a digital systems laboratory class offered to distance learning students. They detailed the way students can participate remotely in such a laboratory as well as the methodology employed for the usability assessment of the laboratory infrastructure (hardware and software) and also outlined the main findings and lessons for learners. This research focused on gaining experience and learning in a digital systems laboratory course attended by a number of distance students as well as performed a usability 
evaluation to ensure the educational quality and students' satisfaction. The evaluation of the laboratory using experimental techniques revealed potential problems and areas for improvement, and future goals are to re-evaluate the laboratory and constantly improve its quality as a part of a summative evaluation plan. Mohamed Tawfik et al. [5] discussed a new technology for performing remote electric and electronic circuit experiments. Virtual Instrument System in Reality (VISIR) has been implemented at the Spanish University for Distance Education (UNED) for new engineering graduate students' distance laboratories. Mohamed Tawfik et al. give a brief update of the project and its implementation, as well as discussed its role in the Electrical and Computer Engineering Department at UNED.

An important objective of the VISIR project was to create a lab community consisting of all of the participant universities and organizations. Consequently, it would be possible to create a grid laboratory where the nodes are online lab workbenches distributed among those universities. Some of the most relevant are merging it with the iLAB project, connecting it to a Learning Management System (LMS), expanding its experiment protocol to include new types and modules of instruments and adopting web services to access the measurement server. Subsequently, the structure of the relay switching matrix could be developed to include more component ranges, instruments, and complex circuits.

Wolbank et al. [6] developed a basic remote-controlled laboratory for experimentation in Electrical Engineering. Their PEMC Web Lab project provides the user with practical experience in Industrial Electronics education. It is designed based on leading ideas and has clear targets. In this project, a laboratory for the course motion control is introduced. First, the description of the system and the two assignments, namely calibration of resolver and current control loop design, were explained. The goal of the experiment, content of the experiment, and verification of the achieved learning outcomes were illustrated in two examples from the area of motion control. Both experiments had concrete learning objectives and evaluation. Although it is too soon to evaluate the overall efficiency of the mentioned educational approach, the first experience is more than encouraging. Juan P. Oliver and Fiorella Haim [7] developed and presented an innovative laboratory methodology for an introductory digital design course. Instead of having traditional lab experiences in which students have to attend classrooms, a "lab at home" concept was proposed. Students would perform real experiments in their own homes using hardware kits specifically developed for this purpose. They would analyze the given problems, design appropriate solutions, and test the actual circuit in a distance learning context. Laboratory learning objectives were analyzed to validate this experience. This research also discusses scalability and different students' work evaluation approaches to facilitate the application of this methodology to other contexts. Moreover, the "lab at home" has been shown to be very suitable for high enrollment courses and is easy to scale, providing actual hardware platforms for students at an affordable cost for the institution. Sanghun Choi and Maryam Saeedifard [8] described a new educational power electronics laboratory that was developed primarily to reinforce experimentally the fundamental concepts presented in a power electronics course. The developed laboratory combines theoretical design, simulation studies, digital control, fabrication, and verification of power- 
electronic circuits based on a set of hardware and software tools. The developed laboratory is a valuable vehicle to enhance active learning and to provide the students with deeper understanding of the design and control of power-electronic circuits. Witold A. Pleskacz et al. [9] described a unique remote laboratory for studying CMOS physical defects meant to be used in advanced courses in the scope of microelectronic design and test. Both the customized measurement equipment and the remote access mechanism were developed in the frame of the European Union project REASON. The core of the equipment is an educational chip that contains manufacturing defects physically implemented into standard digital cells and small logic circuits on the layout level. The chip is supplied with a dedicated plug-and-play measurement box, which provides an interface between the chip and the training software. This measurement kit offers a glimpse into the silicon reality, revealing the behaviour of the most common defects and their influence on the circuit operations. The first run of the Def Sim laboratory exercises was successfully carried out at Tallinn University of Technology, Estonia. Other universities in Central and Eastern Europe are currently integrating laboratory exercises using the Def Sim tool in their educational processes within different courses in the area of VLSI design and testing. Giovanna Oriti, Alexander L. Julian and Daniel Zulaica [10] presented the technology and user-interface development to create a doubly fed induction machine (DFIM) remotely controlled hardware laboratory for DL education. The new laboratory is designed to reinforce the theory learned in class and to verify that modelling and simulations of the laboratory machine are accurate. Students use an oscilloscope to measure currents and voltages and acquire data to build confidence that the simulations are correct. Students' feedback was collected, and it is positive. Future work will include increasing involvement of the student in the drive's control system design, which presently does not meet required expectation.

For engineering education to be available not only on university campuses but also at the students' own location, educational material and the hardware laboratory experiments need to be accessible through the internet. Providing distance learning through a hardware laboratory experience for students is challenging from both logistical and pedagogical points of view. Logistically, the hardware needs to be entirely controllable by a computer. The computer must be a web server providing internet access to the hardware and the student should not be required to have administrator rights to his/her remote computer to access the distance learning laboratory. From a pedagogical point of view, the remote user interface must be simple to understand and operate. It should also provide a meaningful learning experience to add to the theory and modelling that are part of the regular class work.

The undergraduate students studying in the MDT program with majors in Digital Media Technology are required to take a course in Basic Electronics that provides students with theoretical knowledge and practical skills. The laboratory is very important for improving students' skills. The distance laboratory can be an alternative to help students learn and practice more outside the scheduled classroom, which is accessible any time and any place students want to learn.

The National Education Development Plan has a policy for the public and private sectors to educate people to have technological capabilities and knowledge in theory 
and practice. For undergraduate students, studying by using experiments is important to emphasize how to apply skills and transfer them to other subjects and helps students understand the theory and link the knowledge with the principle of theory. The process of learning has five steps: analysis, design, development, application, and evaluation. The problems with the learning via experiments include the content, such as the circuit analysis theory; transferring the knowledge, such as in mathematics; lack of attention to learning; analyses of the students by the teacher; motivation for studying; and interest in the learning process. In operation, the problem is the environment, such as the lack of tools and equipment and the lack of the continuous theory. The students know the result of the experiment is right or wrong because they calculated the result and compared it with the expected result of the experiment. That does not encourage the students because the students learned the principle theory from the teacher and after experiment. The students did not discover the principle theory by themselves, so the students have low achievement learning.

\section{$2 \quad$ Materials and methods}

Figure 1 shows the work flow of the development of the distance laboratory package for teaching basic electronics via cloud computing. The design package contains the actual electronic circuit for the students to able to practice on a simulation of the circuit operation through a LabVIEW program. These operations are connected through the communication video signal via webcams on laptops for the user to learn through the lab experiments. They communicate through a cloud network and storage area connected to the internet, so they need to be online to retrieve information stored for later use.

The cloud is convenient rather than depositing a file in its original format such as images, audio, video, zip files, etc. When files need to be used, they must be downloaded, which is very time consuming. But for file storage with Cloud computing, we can manage those files on our own computer. Therefore, we can access stored files in the Cloud without downloading them. But depending on how the Cloud computing providers offer services and how customers use Cloud computing, the current cloud service providers exist in many forms, including file hosting services and application hosting services. A file hosting and sharing service in Cloud storage is called a "Dropbox," which is easy to use and automatically synchronizes - for example, we store a document file on the Dropbox and we are also able to open the print job immediately as well as pause and save the file. A cloud system will automatically upload and save the files in the Dropbox, while we can run another computer or other device to edit those files. A diagram of how the Distance Laboratory Package for Teaching Basic Electronics works is presented in Fig.1 


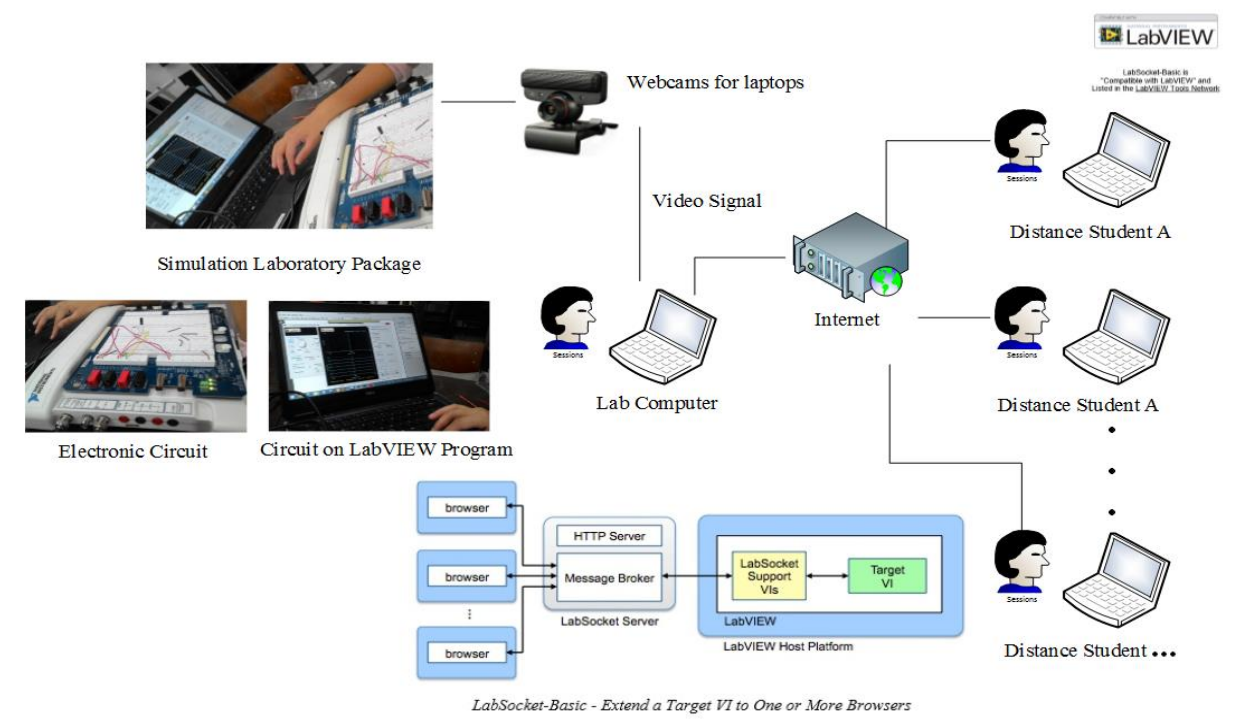

Fig. 1. Diagram of Distance Laboratory Package for Teaching Basic Electronics via Cloud Computing

\subsection{Distance Education}

Distance education, distance learning, learning, or D-Learning is a mode of delivering education and instruction, often on an individual basis, to students who are not physically present in a traditional setting such as a classroom. Distance learning provides "access to learning when the source of information and the learners are separated by time and distance, or both." The University of London was the first university to offer distance learning degrees, establishing its External Program in 1858. This program is now known as the University of London International Program and includes Postgraduate, Undergraduate and Diploma degrees created by colleges such as the London School of Economics, Royal Holloway and Goldsmiths. In the United States William Rainey Harper, the first president of the University of Chicago developed the concept of extended education whereby the research university had satellite colleges of education in the wider community. In 1892, he also encouraged the concept of correspondence school courses to further promote education, an idea that was put into practice by Columbia University.

Although the expansion of the Internet blurs the boundaries, distance education technologies are divided into two modes of delivery: synchronous learning and asynchronous learning. In synchronous learning, all participants are "present" at the same time. In this regard, it resembles traditional classroom teaching methods despite the participants being located remotely. It requires a timetable to be organized. Web conferencing, videoconferencing, educational television, instructional television are examples of synchronous technology, as are direct-broadcast satellite (DBS), internet 
radio, live streaming, telephone, and web-based VoIP. Online meeting software such as Adobe Connect has helped to facilitate meetings in distance learning courses.

The high cost of education affects students in higher education; therefore, distance education may be an alternative to provide some relief. Distance education may be able to save students a considerable amount by removing the cost of transportation. In addition, distance education may be able to save students from the economic burden of high-priced course textbooks. Many textbooks are now available as electronic textbooks, known as e-textbooks, for a reduced price in comparison to traditional textbooks. Also, increasing improvements in technology have resulted in many school libraries having a partnership with digital publishers that offer free course materials, which can help students significantly save on educational costs.

Within the class, students are able to learn in ways that traditional classrooms are not be able to provide. For example, students can review their lessons more than once according to their needs. Students can then manipulate the coursework to fit their learning by focusing more on their weaker topics while breezing through concepts they already have or can easily grasp. When course design and the learning environment are at their optimal conditions, distance education can lead students to higher satisfaction with their learning experiences. Studies have shown that high satisfaction correlates to increased learning. Students who enrolled in distance education with high satisfaction in their online coursework are then intrinsically motivated to learn, which often means their performance in class will improve. For those in a healthcare or mental health distance learning program, online-based interactions have the potential to foster deeper reflections on and discussions of client issues as well as a quicker response to client issues since supervision happens on a regular basis and is not limited to a weekly supervision meeting. This also may contribute to the students feeling a greater sense of support since they have ongoing and regular access to their instructors and other students.

Distance learning may enable students who cannot attend a traditional school setting due to disability or illness, such as decreased mobility and immune system suppression, to get a good education. Distance education may provide equal access regardless of socioeconomic status or income, area of residence, gender, race, age, or cost per student. Applying universal design strategies to distance learning courses as they are being developed (rather than instituting accommodations for specific students on an as-needed basis) can increase the accessibility of such courses to students with a range of abilities, disabilities, learning styles, and native languages. Distance education graduates who never would have been associated with the school under a traditional system may donate money to the school.

Distance learning may also offer a final opportunity for adolescences who are no longer permitted in the general education population due to behavior disorders. Instead of these students having no other academic opportunities, they may continue their education at home and earn their diplomas, offering them another chance to be an integral part of society. 


\subsection{Learning Theories}

Learning theories are conceptual frameworks that describe how information is absorbed, processed, and retained during learning. Cognitive, emotional, and environmental influences, as well as prior experience, all play a part in how understanding, or a world view, is acquired or changed and how knowledge and skills are retained.

Behaviorists look at learning as an aspect of conditioning and advocate for a system of rewards and targets in education. Educators who embrace cognitive theory believe that the definition of learning as a change in behavior is too narrow and prefer to study the learner and, in particular, the complexities of human memory rather than the environment. Humanists emphasize the importance of self-knowledge and relationships in the learning process. Those who advocate constructivism believe that a learner's ability to learn relies to a large extent on what he already knows and understands and that the acquisition of knowledge should be an individually tailored process of construction.

Outside the realm of educational psychology, techniques to directly observe the functioning of the brain during the learning process, such as event-related potential and functional magnetic resonance imaging, are used in educational neuroscience. As of 2012, such studies are beginning to support a theory of multiple intelligences, where learning is seen as the interaction among dozens of different functional areas in the brain, each with its own individual strengths and weaknesses in any particular human learner.

\subsection{Lab view system design software}

LabVIEW software is ideal for any measurement or control system and is the heart of the NI design platform. Integrating all the tools that engineers and scientists need to build a wide range of applications in dramatically less time, LabVIEW is a developmental environment for problem solving, accelerated productivity, and continual innovation.

LabVIEW is a graphical programming platform that helps engineers scale from design to test and from small to large systems. It offers unprecedented integration with existing legacy software, IP and hardware while capitalizing on the latest computing technologies. LabVIEW provides faster and more effective tools to solve today's problems as well as the capacity for future innovations. The NI LabVIEW system design software is at the center of the National Instruments platform. Providing comprehensive tools to build any measurement or control application in dramatically less time, LabVIEW is the ideal developmental environment for innovation, discovery and accelerated results. It combines the power of LabVIEW software with modular reconfigurable hardware to overcome the ever-increasing complexity involved in delivering measurement and control systems on time and under budget. 


\subsection{Distance-Learning Remote Laboratories}

A remote laboratory is defined as a computer-controlled laboratory that can be accessed and controlled externally over some communication medium. For this discussion, a remote laboratory is an experiment, demonstration, or process running locally on a LabVIEW platform with the ability to be monitored and controlled over the Internet from within a Web browser. In the simplest case, the remote laboratory server can be an experiment connected to a computer through a standard interface (DAQ, GPIB, serial, parallel, etc.) with the host computer connected to the Internet. The client can be any computer connected to the Internet running a simple browser. Once connected, the client will see the same front panel as the local host and also have the same program functionality.

\subsection{Electronic Circuit Simulation}

Electronic circuit simulation uses mathematical models to replicate the behaviour of an actual electronic device or circuit. Simulation software allows modelling of circuit operation and is an invaluable analysis tool. Due to its highly accurate modelling capability, many colleges and universities use this type of software for teaching electronics technician and electronics engineering programs. Electronics simulation software engages the users by integrating them into the learning experience. These kinds of interactions actively engage learners in analyzing, synthesizing, organizing, and evaluating content and result in learners constructing their own knowledge.

Simulating a circuit's behaviour before actually building it can greatly improve design efficiency by making faulty designs known and providing insight into the behaviour of electronics circuit designs. In particular, for integrated circuits, the tooling (photo masks) is expensive, breadboards are impractical, and probing the behaviour of internal signals is extremely difficult. Therefore, almost all IC design relies heavily on simulation. The most well-known analog simulator is SPICE. Probably the best known digital simulators are those based on Verilog and VHDL.

Some electronics simulators integrate a schematic editor, a simulation engine, and on-screen waveforms and make "what-if" scenarios easy and instant. They also typically contain extensive model and device libraries. These models typically include IC specific transistor models such as BSIM; generic components such as resistors, capacitors, inductors and transformers; user defined models such as controlled current and voltage sources; or models in Verilog-A or VHDL-AMS. Printed circuit board (PCB) design requires specific models as well, such as transmission lines for the traces and IBIS models for driving and receiving electronics.

\subsection{The instruments used in research}

The instruments used in the current research consisted of prototype research tools, a basic transistor DC biasing circuit and a logic gate circuit on the LabVIEW program. Design and construction of the objectives were set by the behaviour objec- 
tive in the experimental design. When the design was finished, it then performed the following steps.

1. Checked the accuracy and integrity of the experimental.

2. Brought the experiment to try out.

3. Rectified the defect.

4. Identified the performance criteria for the $80 / 80$.

5. Led to the actual try out.

\section{Student assessment tools.}

1. All exams before the experiment.

2. The exam after the experiment.

3. All exams after the experiment.

\subsection{Learning Theories}

\section{Data analysis.}

Questionnaire performance test. The questionnaire was tested on a total of 30 students majoring in Digital Media Technology who had never studied DMT 250 electronic technology for media. The purpose was to use the test results for analysis.

Analysis of questionnaire performance. The test results were scored to determine the Difficulty of the multiple-choice test, Discrimination of the multiple-choice test and the Reliability of the questionnaire. The test with difficulty values between 0.20 0.80 and Discrimination of 0.20 or more was selected and stored as a database. Any test question differing from such criterion will be used to improve new questions and choices before use as a test database. The test was selected from the database for the purpose of making a pretest and a posttest.

A LabVIEW program was created to host experiments for a group of students. Students were divided into 10 groups, each group had two people for each experiment with different sets of experimental kits. When completed, the students took the test. For example, for all 3 experiments, the students tested and retested.

Development of the test: The researcher followed a development process to determine the test quality and performance analysis. The scores from testing during the study and the scores of the exercises were calculated to find the average and percentage. This was then analyzed for process efficiency (E1) and result performance (E2) regarding the development of the test: a distance laboratory package for teaching basic electronics based on Lab VIEW program according to E1 / E2 criterion. The criterion was set at $80 / 80$ by accepting the tolerance.

Progress in the students' learning was analyzed by calculating the pretest scores and posttest scores to determine the difference of each pair of scores and then analyzed using a t-test (Dependent Sample). The criterion for statistical significance was set at a 0.05 level to find progress in learning. The t-test (Dependent Sample) was used to analyze the questionnaire on opinions about the development of the test: a distance laboratory for teaching basic electronics via a LabVIEW program. The scores from a questionnaire on the students' opinions were calculated to find the aver- 
age and standard deviation. Then the results were interpreted according to the set criteria: determining the average of the questionnaire, Standard Deviation.

Tool that model parts. Using the design and distance laboratory package for teaching basic electronics to bachelor's degree students who are studying in the media technology program with a major in Digital Media Technology, KMUTT, the researchers created a process for teaching as follows.

The related documentation. The principles and methods for designing the distance laboratory package for teaching basic electronics from books and research papers from the Internet.

The course materials and the course objectives for the curriculum of the DMT 250 Electronics Technology for Media course for undergraduate students who study in the Media Technology Program with majors in Digital Media Technology, KMUTT, BE 2551 (revised 2553), are as follows.

The objective course

1. To have knowledge and understanding of the rules and basic electronics theories.

2. To have the skills to measure and evaluate the basic electronics circuits.

3. To have a solid introduction and are working with it correctly.

\section{The Course Standards .}

1. To understand the rules and basic electronics circuit theories.

2. To calculate the basic electronics circuits.

3. To connect the test circuit and take measurements.

Course Description: DMT 250 Electronics Technology for Media Course Diploma BE 2551 (Revised 2553) for Media Technology Program majors

Conductivity in metals and semiconductors, PN junction, the properties of equipment made from semiconductors, transistor circuits, operation of Op-Amp circuits and applications, digital logic circuits, Basic Logic Gates, Boolean algebra, and combinational circuits.

\section{Course Analysis Procedure}

Students in the DMT 250 Electronics Technology for Media course BE 2551 (Revised 2553) for Media Technology Program majors spent 3 hours/week for 17 weeks ( 3 credits) focused on the calculated values of electrical tests and measurement skills and then the measurement results were compared with calculations.

Brainstorm Chart Drafting was used to find the details of the course program for the DMT 250 electronics technology for media course related to the basic electronics circuit and the research team of experts and teachers of media technology program majors, which presented a picture of the lessons that should be the overall content. The results of the brainstorming session provided a concrete picture of related ideas.

Concept Chart Drafting is the process of analyzing information from the chart title by brainstorming what might be eliminated. It reveals the knowledge that should be taught in higher level classes after determination of what content experts find to be an appropriate, accurate and comprehensive curriculum. 
Belle Pearl Karun [31] provided a means to analyze information about the course circuit 1 . The study gathered information about careers in the establishment of the rule details. The people who do this work have to be qualified and capable (Task) and know what and how to analyze. There is a hierarchy, as shown in Fig.3

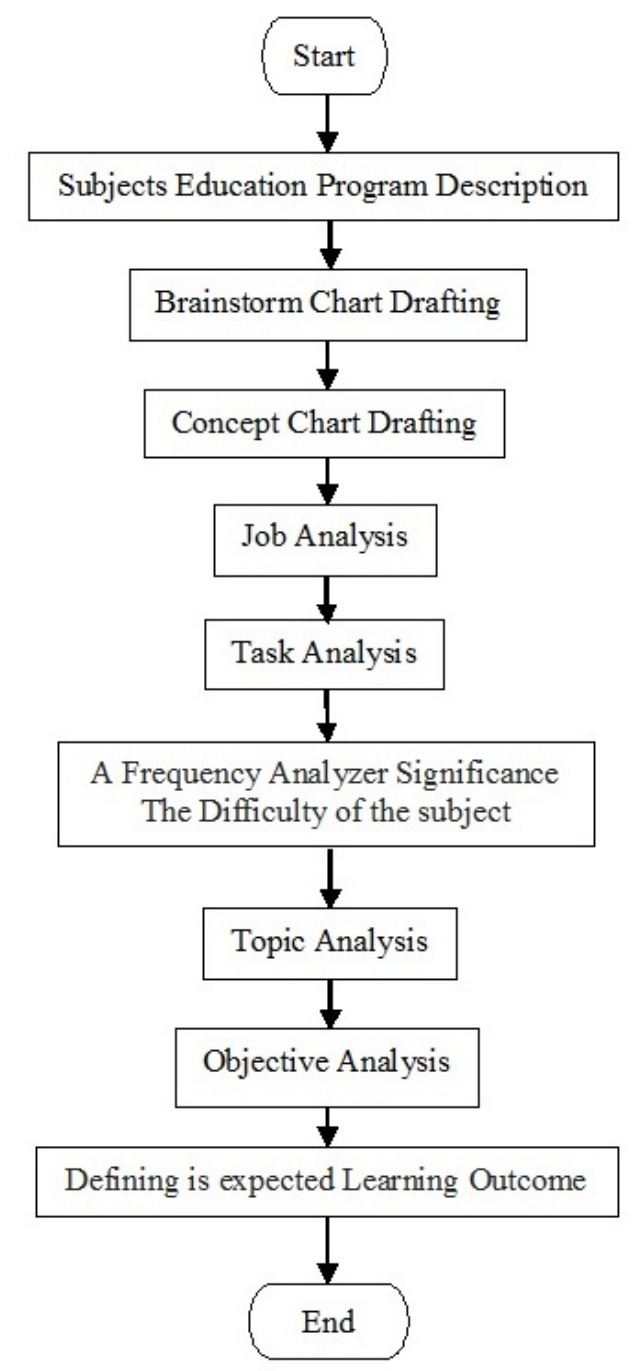

Fig. 2. Course Analysis Procedure

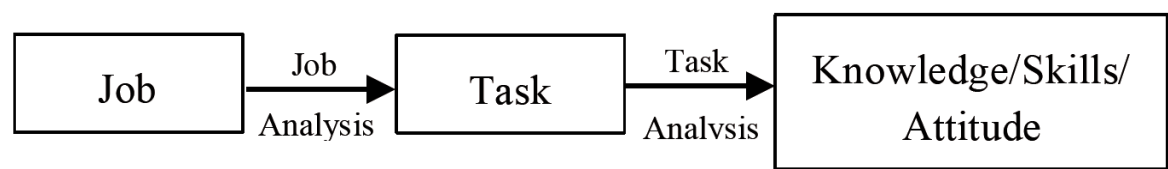

Fig. 3. Schematic representation of hierarchical task analysis 
The researcher adopted content analysis to analyze the DMT 250 electronics technology for the media course to determine details of the main headings and subheadings. The title of the chart Brainstorm (Brainstorm Chart) and chart titled relationship (Concept Chart) were analyzed for the priority of each topic and the order of the subjects. Moving from simple to more complex mattered little.

The principles of Task Analysis were used to analyze the learnability of each subtopic. This provided an analysis of the students' capacity to analyze the main subject, minor subjects, and sub-topics after learning each sub-topic. Each sub-topic was analyzed, resulting in behavioral objectives showing what the students could do.

Topic analysis to find the frequency of the content use as well as the importance and difficulty of the content was concerned with analyzing the topic of the course: the DMT 250 electronics technology dealing with electronic circuits. The objective was to identify the basic content essential for the students to study or solve the problems in the next topics of the course according to the researcher's sorting of the content from easy to difficult. The frequency of the content use as well as the importance and difficulty of the content were analyzed. The researcher and content experts considered sub-topics as the essence of the main topic. Also the objectives of each sub-topic were matched to the objectives of the main topic. The essence was analyzed one by one, leading to the analysis results.

After analyzing the topic related to the frequency of content use and the importance, it was found that the topics of connecting the digital circuit, logic gates, Boolean algebra and combination circuits were crucial, with the difficulty at a high level and a high frequency of the use for learning the next subjects. Therefore, the researcher developed a test of each topic by summarizing the abovementioned topic as basic electronic circuits. After learning with deep understanding, the students would be able to apply knowledge to the next topics well. For this study, the researcher integrated Gate circuit connections into the topic Combination Logic Circuit. Afterwards, the content experts were asked to consider the appropriateness, accuracy and comprehensiveness to develop the most complete content for the topic.

Topic Analysis of basic electronic circuits was aimed at determining the details. Thus, it was advisable to carefully study the relevant information from various sources such as (1) course curriculum, (2) textbooks and documentation, (3) the developer's own experience, and/or (4) the Internet.

Objective Analysis was the determination of the objectives of all of the topics that require the students to change their behavior after learning the topic. The details clearly show how to teach the students to be knowledgeable. The scope of knowledge for each objective determined how the students should apply that knowledge after finishing the topic. Measurable objective levels are as follows.

1. Recall Knowledge

2. Apply Knowledge

3. Transfer Knowledge

4. Define learning objectives for analysis of the course.

5. Determine composition of the development of basic electronics circuits in LabVIEW. 


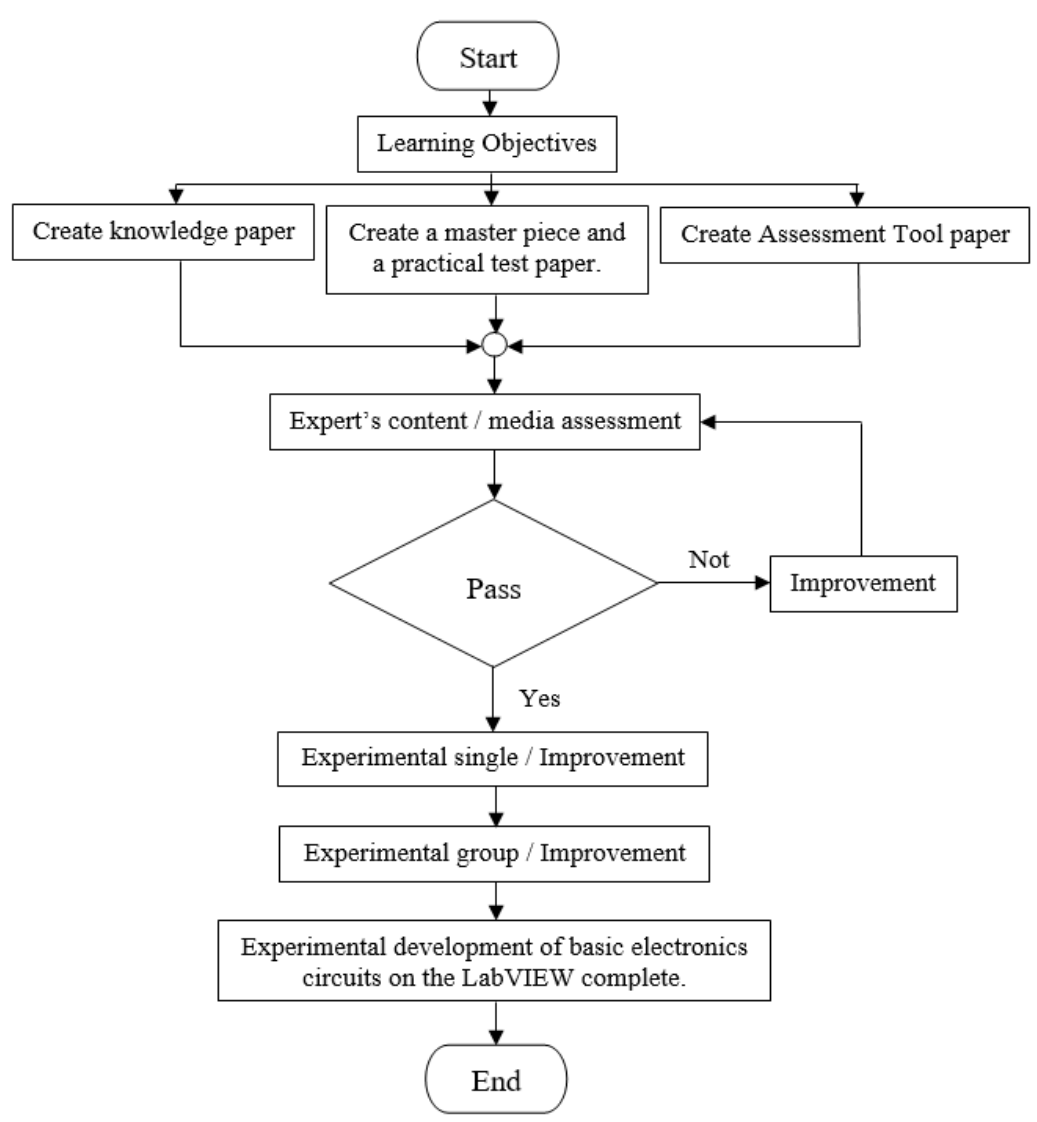

Fig. 4. Process development of basic electronics circuits experiment on the LabVIEW

The students needed to be able to create knowledge with clear illustrations and use of different colors to complement the content digital circuit, logic gates, Boolean algebra and combination circuits.

The students needed to be able to create a master and 4 experiments on digital circuit, logic gates, Boolean algebra and combination circuits prototype specimen trial programs; basic electronics circuits on LabVIEW are designed with ease. Different colors were used to motivate the learners.

\section{$3 \quad$ Results}

A distance laboratory for teaching basic digital logic gate was developed using LabVIEW software as shown in Figures 5-9. Figures 5 and 6 show an OR gate experimental laboratory. Fig. 7 presents an ex-or gate experimental laboratory. Fig. 8 presents the source code in the block diagram of the LabVIEW software. Fig. 9 shows a combinational logic circuit using LabVIEW. 
3.1 Experimental design of the Basic Transistor DC Biasing Circuits on LabVIEW

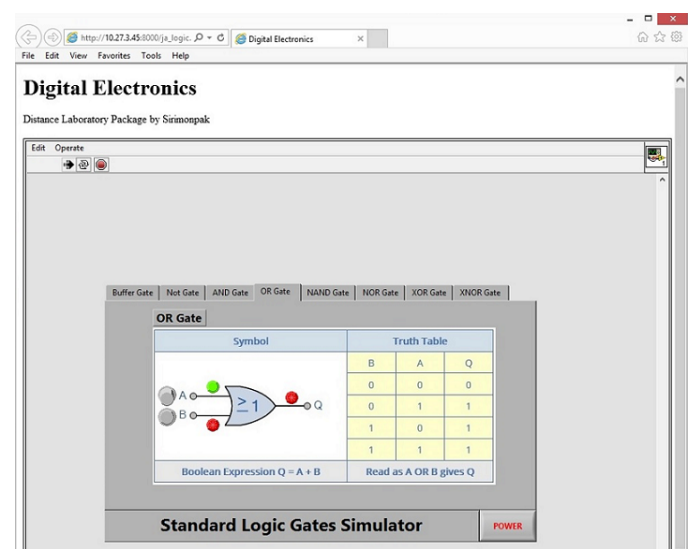

Fig. 5. OR gate simulator: when input $A=$ " 0 ", input $B=$ " 1 " and output $Q=$ " 1 "

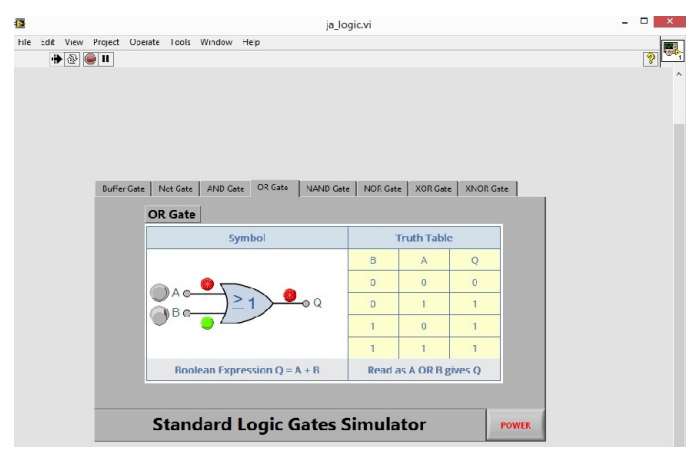

Fig. 6. OR gate simulator: when input $A=$ " 1 ", input $B=$ "0" and output $\mathrm{Q}=$ " 1 "

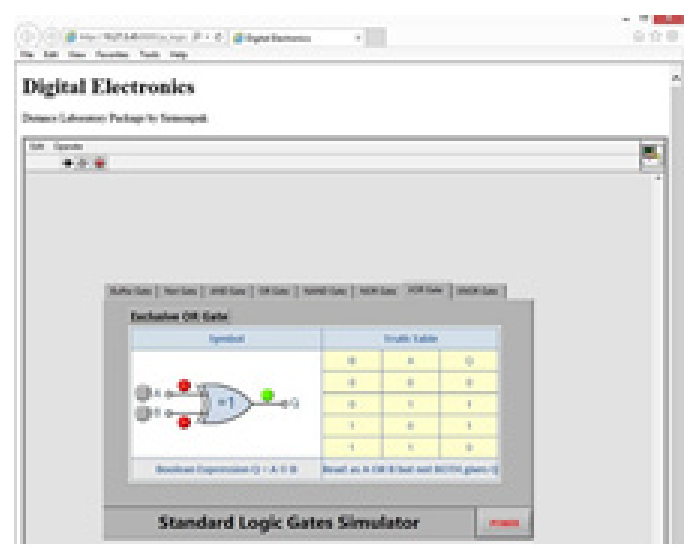

Fig. 7. EX-OR gate simulator: when input $A=$ " 1 ", input $B=$ " 1 " and output $\mathrm{Q}=$ " $0 "$ 
Paper-Design and Development of Distance Laboratory Package for Teaching Basic Electronics via ...

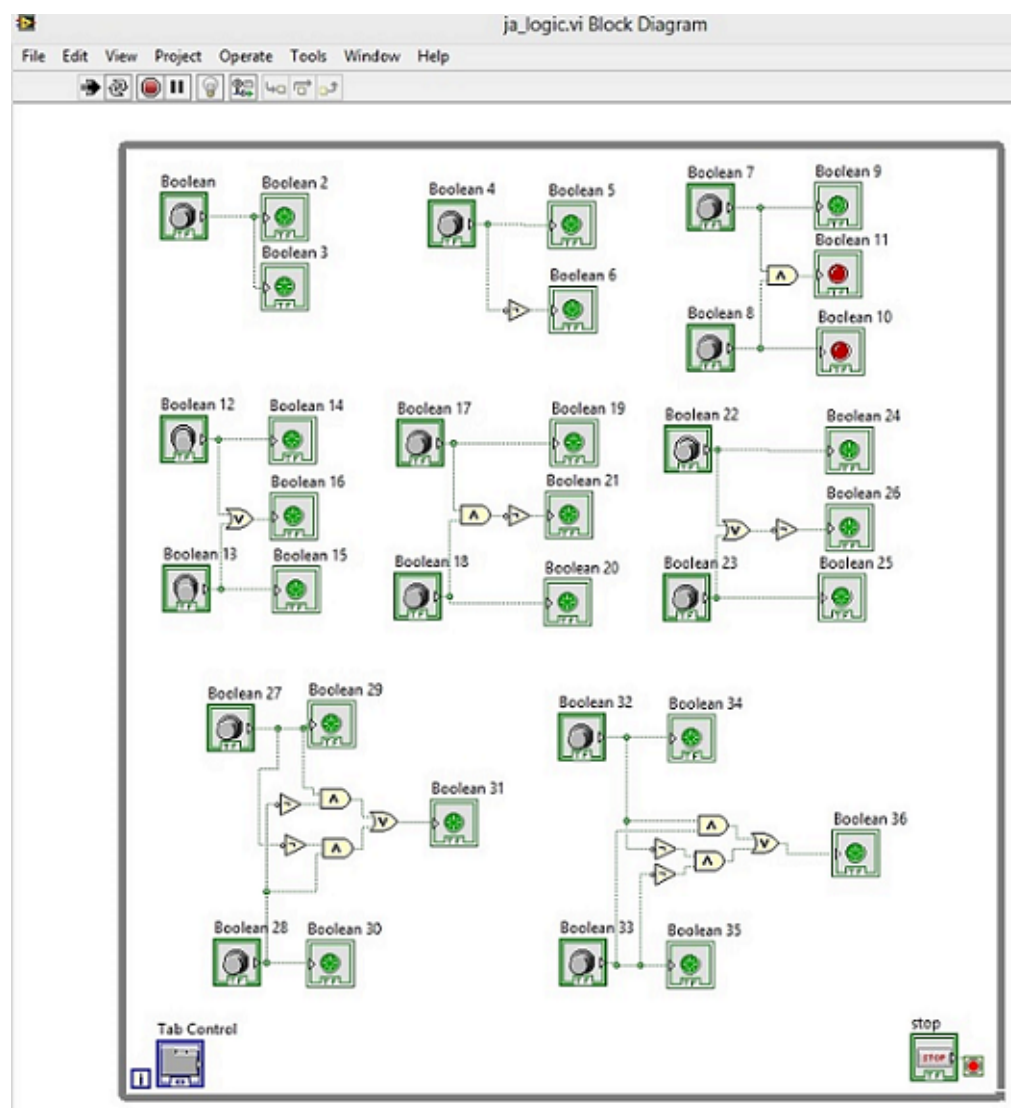

Fig. 8. Block diagram of package in LabVIEW software

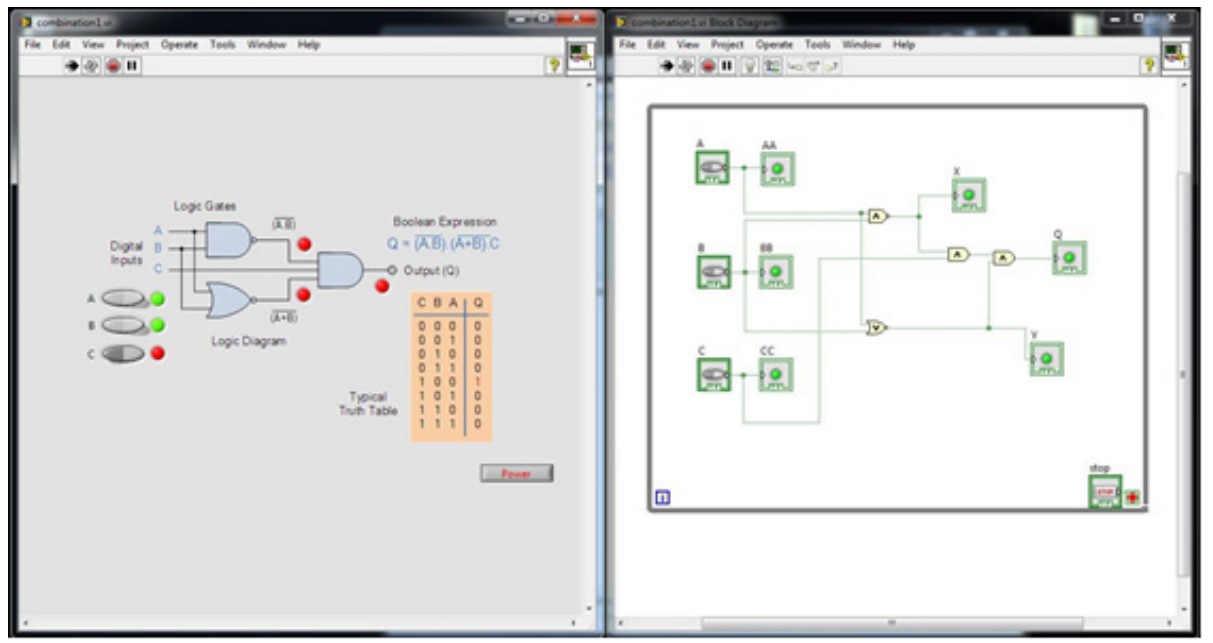

Fig. 9. Combination Logic Circuit on LabVIEW Program 
There are 4 parts in the analysis of data.

1. The analysis results of the consistency between the tests and behavior objective. Given the experimental tests of students who passed the basic transistor DC biasing circuit units course, the results of the difficulty are 0.51 , the classification is 0.47 , and the confidence is 0.85 .

2. nalysis of the efficiency of the experimental results for bachelor degree-level students studying in the Media Technology Program with majors in Digital Media Technology at King Mongkut's University of Technology Thonburi show they had a standard efficiency criteria of $80 / 80$.

3. The analysis results of the academic progress of the students show the average score after studying was higher than the average of the scores before studying. The calculated value is higher than the significance level of 0.05 .

4. Analysis of the results of the student questionnaire about the experimental design for undergraduate students studying in the Media Technology Program with majors in Digital Media Technology at King Mongkut's University of Technology Thonburi show the students had a favorable opinion of the experiments

\section{Discussion}

This article presented the Design and Development of a Distance Laboratory Package for teaching undergraduate students basic electronics through experimental learning via a LabVIEW program. The researcher designed the research process, content analysis, research purpose, and learning activities. For example, some tests (pre-tests and post-tests) showed the knowledge development and impact on students' learning. This research was tested and carried out to help us know that students can learn by themselves through basic electronics experiments. This has been a great inspiration for students and has made them want to learn more about the basic electronics experimental process. A distance laboratory package can help improve the learning and teaching in a class.

\section{$5 \quad$ References}

[1] Distance education, (2013), In Wikipedia. Modified on 10 October, http://en.wikipedia.org /wiki/Distance education.

[2] Learning theories, (2013), In Wikipedia. Modified on 11 October, http://en.wikipedia.org /wiki/ Learning_theories.

[3] Electronic circuit simulation, (2013), In Wikipedia. Modified on 21 August, http://en.wiki pedia.org/wiki/ Learning theories.

[4] Juan P. Oliver and Fiorella Haim, (2009), Lab at Home: Hardware Kits for a Digital Design Lab, IEEE Journals \& Magazines Transactions on Education, Vol. 52. NO. 1, February, pp. 46-51.

[5] Sanghun Choi, and Maryam Saeedifard, (2012), An Educational Laboratory for Digital Control and Rapid Prototyping of Power Electronic Circuits, IEEE Journals \& Magazines 
Paper-Design and Development of Distance Laboratory Package for Teaching Basic Electronics via ...

Transactions on Education, Vol. 55. NO. 2, May, pp. 263-270. https://doi.org/10.1109/ TE.2011.2169066

[6] Arporn Jaithiang, (2007), Principle of Teaching, Odian Store, The fourth press.

[7] Black Beverly and others, Guidebook for Teaching Labs for University of Michigan Graduate Student Instructor, Center for Research on Learning andTeaching: http://www.erit.umich.edu/gsis lab_guidebook.html.

[8] Chusak Plienpoo, Workshop and Laboratory Instruction System, Faculty of Industrial Education and Technology, King Mongkut's University of Technology, Thonburi.

[9] Kanda Poonlabtavee, (1985), Measurement and Evaluate, Technical Education and Technology department, Faculty of Technical Education and Science.

[10] Luan Saiyod and Angkana Saiyod, (1987), Educational Research Technical the 5th Bangkok, Suviriyasarn.

[11] Robert L. Boylestad, Louis Nashelsky. Electronic Devices and Circuit Theory, (2009), Pearson Education New Jersey.

[12] Nektarios Kostaras, Michalis Xenos and Athanassios N. Skodras, (2011), Evaluating Usability in a Distance Digital Systems Laboratory Class, IEEE Journals \& Magazines Transactions on Education, Vol. 54. NO. 2, May, pp. 308-313.

[13] Mohamed Tawfik, Elio Sancristobal, Sergio Martin, Charo Gil, Pablo Losada, Gabriel Diaz and Manuel Castro, (2011), Remote Laboratories for Electrical \& Electronic Subjects in New Engineering Grades, IEEE Promotion and Innovation with New Technologies in Engineering Education, 5-6 May 2011.

[14] T.M. Wolbank, P.Bauer, P. Macheiner and M. Vogelsberger, (2008), Distance Laboratory for Teaching Industrial Electronics, IEEE Journals \& Magazines Transactions on Education, pp. 3497-3502.

[15] Juan P. Oliver and Fiorella Haim, (2009), Lab at Home: Hardware Kits for a Digital Design Lab, IEEE Journals \& Magazines Transactions on Education, Vol. 52. NO. 1, February, pp. 46-51.

[16] Sanghun Choi, and Maryam Saeedifard, (2012), An Educational Laboratory for Digital Control and Rapid Prototyping of Power Electronic Circuits, IEEE Journals \& Magazines Transactions on Education, Vol. 55. NO. 2, May, pp. 263-270. https://doi.org/10.1109/ TE.2011.2169066

[17] Witold A. Pleskacz, Viera Stopjakova, Tomasz Borejko, Artur Jutman, Member and Andrzej Wałkanis, DefSim: A Remote Laboratory for Studying Physical Defects in CMOS Digital Circuits, IEEE Journals \& Magazines Transactions on Industrial Electronics, Vol. 55. NO. 6, June, pp. 2405-2415.

[18] Giovanna Oriti, Alexander L. Julian and Daniel Zulaica, (2014), Doubly Fed Induction Machine Drive Hardware Laboratory for Distance Learning Education, IEEE Journals \& Magazines Transactions on Power Electronics, Vol. 29. No.1, January, pp. 440-448. https://doi.org/10.1109/TPEL.2013.2252629

[19] Mohamed Tawfik, Elio San Cristobal, Alberto Pesquera, Rosario Gil, Sergio Martin, Gabriel Diaz, Juan Peire, Manuel Castro, Pablo Orduna and Javier Garcia-Zubia, (2012), Putting Fundamentals of Electronic Circuits Practices Online, IEEE Journals \& Magazines Transactions on Education, pp. 117-121.

[20] P.Bauer, (2008), Distance Laboratory for Teaching Power Electronics-Part II DC-AC, IEEE International Conference on Control and Modeling for Power Electronics. https://doi.org/10.1109/COMPEL.2008.4634704

[21] S. Chopra, P. Bauer, (2011), Distance Laboratory for Teaching Electrical Characteristics Measurement and Maximum Power Point Tracking of a Photovoltaic Module, IEEE Journals \& Magazines Transactions on Education, pp. 22-27. 
[22] Jing Zhang, Yu Mei and Ming-li Li, (2012), Construction of the engineering experimental teaching system for remote education based on the model analysis, IEEE International Conference on Consumer Electronics Communications and Networks (CECNet), 2012 2nd, No. 1734. Pp. 1728-1734.

[23] Unn. Doris, Karlsen Baeck, (2009), From a distance-How Norwegian parents experience their encounters with school, Science Direct International Journal of Educational Research, 48 No. pp. 342-351.

[24] E. San Cristobal Ruiz, M. Tawfik, S. Martin, R. Gil, A. Pesquera, F. Garcia, V. J. Rodriguez, F. Mur, M.J. Albert, C. Perez, G Diaz and M. Castro, (2013), Design Development and Implementation of Remote Laboratories in Distance Electronics Control and Computer Subjects, IEEE International Conference on Remote Engineering and Virtual Instrumentation (REV), 2013 10th.

[25] Sanghun Choi, and Maryam Saeedifard, (2012), An Educational Laboratory for Digital Control and Rapid Prototyping of Power Electronic Circuits, IEEE Transactions on Education, Vol. 55. NO. 2, May, pp. 263-270. https://doi.org/10.1109/TE.2011.2169066

[26] Nektarios Kostaras, Michalis Xenos, and Athanassios N. Skodras, (2011), Evaluating Usability in a Distance Digital Systems Laboratory Class, IEEE Transactions on Education, Vol. 54. NO. 2, May, pp. 308-313. https://doi.org/10.1109/TE.2010.2054096

[27] Ben Hanson, Peter Culmer, Justin Gallagher, Kate Page, Elizabeth Read, Andrew Weightman, and Martin Levesley, (2009), ReLOAD: Real Laboratories Operated at a Distance, IEEE Journals \& Magazines Transactionson Learning Technologies, Vol. 2. NO. 4, October-December, pp. 331-341. https://doi.org/10.1109/TLT.2009.35

[28] Mitalkumar G. Kanabar, Tarlochan S. Sidhu, and Mohammad R. D. Zadeh, (2011), Laboratory Investigation of IEC 61850-9-2-Based Busbar and Distance Relaying with Corrective Measure for Sampled Value Loss/Delay, IEEE Journals \& Magazines Transactions on Power Delivery, Vol. 26. No. 4, October, pp.2587-2595. https://doi.org/10.1109/TPWRD. $\underline{2011.2159033}$

[29] I. Santana, M. Ferre, E. Izaguirre, R. Aracil and L. Hernandez, (2013), Remote Laboratories for Education and Research Purposes in Automatic Control Systems, IEEE Journals \& Magazines Transactions on Industrial Informatics, Vol. 9. No. 1, February, pp. 547-556. https://doi.org/10.1109/TII.2011.2182518

[30] Shaphan R. Jernigan, Yusef Fahmy and Gregory D. Buckner, (2009), Implementing a Remote Laboratory Experience Into a Joint Engineering Degree Program: Aerodynamic Levitation of a Beach Ball, IEEE Journals \& Magazines Transactions on Education, Vol.

[31] 52. No. 2, May, pp. 205-213.

[32] Andreja Rojko, Darko Hercog and Karel Jezernik, (2010), Power Engineering and Motion Control Web Laboratory: Design, Implementation, and Evaluation of Mechatronics Course, IEEE Journals \& Magazines Transactions on Industrial Electronics, Vol. 57. No. 10, October, pp. 3343-3354. https://doi.org/10.1109/TIE.2009.2031189

[33] Nuno Sousa, Gustavo R. Alves, and Manuel G. Gericota, (2010), An Integrated Reusable Remote Laboratory to Complement Electronics Teaching, IEEE Journals \& Magazines Transactions on Learning Technologies, Vol. 3. No. 3, July-September, pp. 265-271. https://doi.org/10.1109/TLT.2009.51

[34] Coia Ferrater-Simon, Lluis Molas-Balada, Oriol Gomis-Bellmunt, Noelia LorenzoMartinez, Oriol Bayo-Puxan and Roberto Villafafila-Robles, (2009), A Remote Laboratory Platform for Electrical Drive Control Using Programmable Logic Controllers, IEEE Journals \& Magazines Transactions on Education, Vol. 52. No. 3, August, pp. 425-435. https://doi.org/10.1109/TE.2008.930095 
Paper-Design and Development of Distance Laboratory Package for Teaching Basic Electronics via ...

[35] L. Agostinho, A.F. Farias, L.F. Faina, E.G. Guimaraes, P.R.S.L. Coelho and E. Cardozo, (2010), NetLab Web Lab: A Laboratory of Remote Experimentation for the Education of Computer Networks Based in SOA, IEEE Journals \& Magazines Transactions on Revista IEEE Latin America, Vol. 8. No. 5, September, pp. 597-604. https://doi.org/10.1109/TLA.2010.5623514

\section{Authors}

Sirimonpak Suwannakhun (corresponding author) is Doctor of Philosophy, Program Learning Innovation and Technology, at Faculty of Industrial Education and Technology, King Mongkut's University of Technology Thonburi (KMUTT), Bangkok, Thailand (sirimonpak.sut@kmutt.ac.th, http://orcid.org/0000-0002-6130-6471).

Tanes Tanitteerapan is with the Department of Electrical Technology Education, Faculty of Industrial Education and Technology, King Mongkut's University of Technology Thonburie

Article submitted 08 April 2017. Published as resubmitted by the authors 16 May 2017. 